\title{
A RADIAL AVERAGING TRANSFORMATION, CAPACITY AND CONFORMAL RADIUS
}

\author{
BY MOSHE MARCUS
}

Communicated by F. W. Gehring, October 18, 1971

Introduction. Let $\mathscr{D}=\left\{D_{1}, \ldots, D_{n}\right\}$ be a family of domains in the plane, containing the origin. We define a radial averaging transformation $\mathscr{R}_{A}$ on $\mathscr{D}$ by which we obtain a starlike domain $D^{*}$. When $\mathscr{D}$ is such that the domains $D_{1}, \ldots, D_{n}$ are obtained from a fixed domain $D$ by rotation or reflexion, $\mathscr{R}_{A}$ becomes a radial symmetrization. One of the results we present is an inequality relating the conformal radius of $D^{*}$ to the conformal radii of $D_{1}, \ldots, D_{n}$ at the origin. This result includes, as particular. cases, the radial symmetrization results of Szegö [6] (for starlike domains), Marcus [4] (for general domains) and Aharonov and Kirwan [1]. The inequality for the conformal radii is obtained via an inequality for.conformal capacities. A number of applications in the theory of functions is described.

1. Let $M$ be the half strip $\{(x, y) \mid 0<x<1,0<y\}$. We shall say that a function $f$ is of class $\bar{B}(M)$ if

(i) $f$ is continuous in $\bar{M}(=$ closure of $M)$;

(ii) $0 \leqq f \leqq 1$ in $M$;

(iii) the set $\Omega_{1}=\{(x, y) \mid f(x, y)<1\} \cap M$ is bounded;

(iv) on any half line $\left\{x=x_{0}\right\} \cap \bar{M}, f$ assumes every value $\lambda, 0<\lambda<1$, at least once, but not more than a finite number of times;

(v) $f \in C^{1}(\bar{\Omega}(f))$, where $\Omega(f)=\{(x, y) \mid 0<f(x, y)<1\} \cap M$;

(vi) for any line $x=x_{0}, 0 \leqq x_{0} \leqq 1$, the set of points on $\left\{x=x_{0}\right\} \cap \bar{\Omega}(f)$ where $\partial f / \partial y=0$ is finite.

If $f \in \bar{B}(M)$ we denote

$$
\begin{array}{cl}
\Omega_{\lambda}(f)=\{(x, y) \mid f(x, y)<\lambda\} \cap M & (0<\lambda \leqq 1), \\
\Omega_{0}(f)=\{(x, y) \mid f(x, y)=0\} \cap M . & \\
l\left(x_{0}, \lambda ; f\right)=\operatorname{meas}\left(\left\{x=x_{0}\right\} \cap \Omega_{\lambda}(f)\right) & (0 \leqq \lambda \leqq 1),
\end{array}
$$

where the measure is the linear Lebesgue measure. We note that $l\left(x_{0}, \lambda ; f\right)$ is a strictly monotonic increasing function of $\lambda, 0 \leqq \lambda \leqq 1$.

We now introduce

Definition 1.1. Let $\mathscr{F}=\left\{f_{1}, \ldots, f_{n}\right\} \subset \bar{B}(M)$ and let $A=\left\{a_{1}, \ldots, a_{n}\right\}$ be a set of positive numbers such that $\sum_{j=1}^{n} a_{j}=1$. Denote

AMS 1970 subject classifications. Primary 30A44, 30A36; Secondary 31A15, 30A32.

Key words and phrases. Conformal capacity, Dirichlet integral, conformal radius, radial symmetrization, complex analytic functions in the unit disk. 


$$
\begin{gathered}
l^{*}(x, \lambda)=\sum_{j=1}^{n} a_{j} l\left(x, \lambda ; f_{j}\right) \\
\Omega_{\lambda}^{*}=\Omega_{\lambda}^{*}(\mathscr{F}, A)=\left\{(x, y) \mid 0<y<l^{*}(x, \lambda)\right\} \cap M \quad(0<\lambda \leqq 1), \\
\Omega_{0}^{*}=\Omega_{0}^{*}(\mathscr{F}, A)=\left\{(x, y) \mid 0 \leqq y \leqq l^{*}(x, 0)\right\} \cap M, \\
\Omega^{*}=\Omega^{*}(\mathscr{F} ; A)=\Omega_{1}^{*}-\Omega_{0}^{*} .
\end{gathered}
$$

Then the linear averaging transformation $\mathscr{L}_{A}$ on $\mathscr{F}$ is defined as follows:

$$
\begin{aligned}
f^{*}(x, y)=\mathscr{L}_{A}(\mathscr{F})=0, & \text { if }(x, y) \in \Omega_{0}^{*}, \\
& =\lambda, \quad \text { if } y=l^{*}(x, \lambda), 0<\lambda<1, \\
& =1, \quad \text { if }(x, y) \in M-\Omega_{1}^{*} .
\end{aligned}
$$

The following two results are the main steps in the derivation of the main theorems.

Lemma 1.1. Let $\mathscr{F}$ and $A$ be as in Definition 1.1. Then $f^{*}$ is uniformly Lipschitz in $M$.

THEOREM 1.1. Let $\mathscr{F}$ and $A$ be as in Definition 1.1. Let $G(t)$ be a function defined for $t \geqq 0$ such that $G(t)$ is continuous, convex and nondecreasing. Then, with the notations introduced above, we have

$$
\iint_{\Omega^{*}} G\left(\left(1+\left|\nabla f^{*}\right|^{2}\right)^{1 / 2}\right) d x d y \leqq \sum_{j=1}^{n} a_{j} \iint_{\Omega\left(f_{j}\right)} G\left(\left(1+\left|\nabla f_{j}\right|^{2}\right)^{1 / 2}\right) d x d y
$$

where $\Omega\left(f_{j}\right)=\Omega_{1}\left(f_{j}\right)-\Omega_{0}\left(f_{j}\right)$.

COROLlary.

$$
\iint_{\Omega^{*}}\left|\nabla f^{*}\right|^{p} d x d y \leqq \sum_{j=1}^{n} a_{j} \iint_{\Omega\left(f_{j}\right)}\left|\nabla f_{j}\right|^{p} d x d y \quad(1 \leqq p) .
$$

Note that the left side of (1.6) is meaningful because of Lemma 1.1.

2. A condenser in the plane is a system $C=\left(\Omega, E_{0}, E_{1}\right)$ where $\Omega$ is a domain and $E_{0}, E_{1}$ are disjoint closed sets whose union is the complement of $\Omega$. We shall assume also that $E_{0}$ is compact and $E_{1}$ unbounded. An alternative notation for $C$ will be $C=\left(D, E_{0}\right)$ where $D=\Omega \cup E_{0}$.

If $\Omega$ satisfies the segment property (i.e., for any point $P$ on the boundary of $\Omega$ there exists a segment $\overline{P P}^{\prime}$ lying outside $\Omega$ ), there exists a unique function $\omega$, called the potential function of $C$, such that $\omega$ is harmonic in $\Omega$ and continuous in the extended plane and such that $\omega \equiv 0$ on $E_{0}$ and $\omega \equiv 1$ on $E_{1}$. In this case the conformal capacity of $C$ may be defined by 


$$
I(C)=\operatorname{Dir}_{\Omega}[\omega] \equiv \iint_{\Omega}|\nabla \omega|^{2} d x d y .
$$

We now introduce

Definition 2.1. Let $\mathscr{D}=\left\{D_{1}, \ldots, D_{n}\right\}$ be a family of open sets in the complex plane $z$. Suppose that the closed disk $\left|z-z_{0}\right| \leqq \rho$ (for some $\rho>0)$ is contained in $\bigcap_{j=1}^{n} D_{j}$. Let

$$
\begin{aligned}
K_{j}^{\rho}(\phi) & =\left\{r \mid z=z_{0}+r e^{i \phi} \in D_{j}, \rho<r<\infty\right\} \quad(0 \leqq \phi<2 \pi) ; \\
l_{j}^{\rho}(\phi) & =\int_{K_{j}^{\rho}(\phi)} \frac{d r}{r} \text { and } \quad R_{j}(\phi) \equiv R\left(\phi ; D_{j} ; z_{0}\right)=\rho \exp l_{j}^{\rho}(\phi) .
\end{aligned}
$$

(Note that $R_{j}(\phi)$ does not depend on $\rho$.) Set

$$
R^{*}(\phi)=\prod_{j=1}^{n} R_{j}(\phi)^{a_{j}}
$$

$$
D^{*}=\mathscr{R}_{A}\left(\mathscr{D} ; z_{0}\right)=\left\{z=z_{0}+r e^{i \phi} \mid 0 \leqq r<R^{*}(\phi), 0 \leqq \phi<2 \pi\right\} .
$$

We shall say that $\mathscr{R}_{A}$ is a radial averaging transformation on $\mathscr{D}$ with center $z_{0}$.

If $\left\{C_{j}\right\}_{j=1}^{n}$ is a family of condensers, $C_{j}=\left(\Omega_{j}, E_{0, j}, E_{1, j}\right)=\left(D_{j}, E_{0, j}\right)$ where $\bigcap_{1}^{n} E_{0, j} \supseteq\left\{\left|z-z_{0}\right| \geqq \rho\right\}$ we define

$$
C^{*}=\mathscr{R}_{A}\left(\left\{C_{j}\right\} ; z_{0}\right)=\left(D^{*}, E_{0}^{*}\right)
$$

where $D^{*}=\mathscr{R}_{A}\left(\left\{D_{j}\right\} ; z_{0}\right)$ and $E_{0}^{*}=\mathscr{R}_{A}\left(\left\{E_{0, j}\right\} ; z_{0}\right)$. ( $E_{0}^{*}$ is defined in the same way as $D^{*}$ except that in (2.5) we have $0 \leqq r \leqq R^{*}(\phi)$.)

We can now formulate the main result.

THEOREM 2.1. Let $\left\{C_{1}, \ldots, C_{n}\right\}$ be a family of condensers as above, and let $C^{*}$ be defined as in (2.6). Suppose that the domains $\Omega_{1}, \ldots, \Omega_{n}$ have the segment property. Then

$$
I\left(C^{*}\right) \leqq \sum_{1}^{n} a_{j} I\left(C_{j}\right)
$$

The proof is based on Theorem 1.1. We may assume that $z_{0}=0$ and $\rho=1$. We map the domain $|z|<1$, cut along the positive real axis, by $w=\ln z$ onto the half strip $0<u<\infty, 0<v<2 \pi(w=u+i v)$. Let $\omega_{j}$ be the potential function of $C_{j}$. Denote by $f_{j}(u, v)$ the function $\omega_{j}$ represented in $(u, v)$ coordinates. Then we apply Theorem 1.1 (or, more precisely, inequality (1.7) with $p=2)$ to $\mathscr{F}=\left\{f_{1}, \ldots, f_{n}\right\}$ in the strip mentioned above. 
If $D$ is a domain in the plane and $z_{0} \in D$, denote by $r\left(z_{0}, D\right)$ the conformal (or inner) radius of $D$ at $z_{0}$. (For definition and properties see for instance Hayman [3, pp. 78-83].) Using a theorem of Pólya and Szegö [5] on the relation between conformal radius and conformal capacity and Theorem 2.1 we obtain

THEOREM 2.2. Let $\mathscr{D}=\left\{D_{1}, \ldots, D_{n}\right\}$ be a family of domains such that $z_{0} \in \bigcap_{1}^{n} D_{j}$. Let $D^{*}=\mathscr{R}_{A}\left(\mathscr{D} ; z_{0}\right)($ Definition 2.1). Then

$$
\prod_{j=1}^{n} r\left(z_{0}, D_{j}\right)^{a_{j}} \leqq r\left(z_{0}, D^{*}\right) \text {. }
$$

As a first application of Theorem 2.2 we obtain the following symmetrization result :

THEOREM 2.3. Let $f(z)=a_{1} z+a_{2} z^{2}+\cdots$ be an analytic function in the unit disk $|z|<1$. Let $D$ be the image of $|z|<1$ by $w=f(z)$. Let $A=\left\{a_{j}\right\}_{1}^{n}$ be a set of positive numbers such that $\sum_{1}^{n} a_{j}=1$, let $\left\{\alpha_{j}\right\}_{1}^{n}$ be a set of integers $\left(\alpha_{j} \neq 0\right)$ and let $\left\{\beta_{j}\right\}_{1}^{n}$ be an arbitrary set of real numbers.

If $R(\phi)=R(\phi ; D ; 0)($ see $(2.3))$ set

$$
\begin{aligned}
R^{*}(\phi) & =\prod_{j=1}^{n} R\left(\alpha_{j} \phi+\beta_{j}\right)^{b_{j}}, \quad \text { where } b_{j}=a_{j} /\left|\alpha_{j}\right| \\
D^{*} & =\left\{w=\sigma e^{i \phi} \mid 0 \leqq \sigma<R^{*}(\phi), 0 \leqq \phi<2 \pi\right\} .
\end{aligned}
$$

Then

$$
\left|a_{1}\right| \leqq r(0, D) \leqq r\left(0, D^{*}\right)^{1 / b}, \quad \text { where } b=\sum_{1}^{n} b_{j} .
$$

Theorem 2.3 includes as particular cases the radial symmetrization results of Szegö [6], Marcus [4] and Aharonov and Kirwan [1].

We bring now two applications of the preceding theorems.

TheOREM 2.4. Let $f(z)$ and $D$ be as in Theorem 2.3. Denote

$$
D_{t}=\left\{w=\sigma e^{i \phi} \mid 0 \leqq \sigma<R(\phi)^{t}, 0 \leqq \phi<2 \pi\right\} \quad(0<t<1),
$$

where $R(\phi)=R(\phi ; D ; 0)$. Then

$$
\left|a_{1}\right| \leqq r(0, D) \leqq r\left(0, D_{t}\right)^{1 / t} .
$$

THEOREM 2.5. Let $f(z)=z+a_{2} z^{2}+\cdots$ and $D$ be as in Theorem 2.3. Let $R^{*}(\phi)$ be defined as in (2.9). Suppose that $R^{*}(\phi) \leqq M \leqq \infty, 0 \leqq \phi<2 \pi$. Suppose also that for some set of $m$ rays issuing from the origin, with arguments $\phi_{1}, \ldots, \phi_{m}$ we have

$$
\sup _{1 \leqq j \leqq m} R^{*}\left(\phi_{j}\right)=K
$$


Let $D_{0}$ be the disk $|w|<M$ (the entire plane if $M=\infty$ ) cut along the rays $w=\sigma e^{i \phi_{j}}, K_{0} \leqq \sigma<M, j=1, \ldots, m$, where $K_{0}$ is so chosen that $r\left(0, D_{0}\right)$ $=1$. (It follows from our assumptions that $M \geqq 1$.) Then $K_{0} \leqq K$.

Theorem 2.5 implies a number of special "covering" theorems such as Theorem 5 and 6 of Marcus [4] and Theorem 4.2 of Aharonov and Kirwan [1].

A complete presentation of the results described in this note and additional applications will appear elsewhere. We mention also that a discussion of radial averaging transformations with metrics of the form $g(r) d r d \phi$ is given in [2].

\section{REFERENCES}

1. D. Aharonov and W. E. Kirwan, A method of symmetrization and applications, Technical Report \# 70-179, University of Maryland, College Park, Md.; Trans. Amer. Math. Soc. 163 (1972), 369-377.

2. C. Bandle and M. Marcus, Radial averaging transformations with various metrics, Technical Report \# 71-32, Carnegie-Mellon University, Pittsburg, Pa.

3. W. K. Hayman, Multivalent functions, Cambridge Tracts in Math. and Math. Phys., no. 48, Cambridge Univ. Press, New York, 1958. MR 21 \# 7302.

4. M. Marcus, Transformations of domains in the plane and applications in the theory of functions, Pacific J. Math. 14 (1964), 613-626. MR 29 \# 2382.

5. G. Pólya and G. Szegö, Isoperimetric inequalities in mathematical physics, Ann. of Math. Studies, no. 27, Princeton Univ. Press, Princeton, N.J., 1951. MR 13, 270.

6. G. Szegö, On a certain kind of symmetrization and its applications, Ann. Mat. Pura Appl. (4) 40 (1955), 113-119. MR 17, 1074.

Department of Mathematics, Carnegie-Mellon University, Pittsburgh, PennsylVANIA 15213

Current address: Department of Mathematics, Technion, Haifa, Israel. 